\title{
Palmitate impairs cytokinesis associated with RhoA inhibition
}

Cell Research (2010) 20:492-494. doi: 10.1038/cr.2010.33; published online 16 March 2010

\section{Dear Editor,}

Excess fatty acid accumulation in non-adipose tissues results in lipotoxicity, which has been implicated in the pathogenesis of metabolic diseases such as obesity and diabetes [1]. A number of tissue culture systems have been used to study lipotoxicity by supplementation of culture media with palmitate, which is a major saturated free fatty acid in human plasma and has been reported to induce apoptosis in various cell types [2]. Here we report that palmitate causes formation of binucleate cells as a consequence of cytokinetic impairment. Our results reveal a novel toxic effect of palmitate on cell division and extend the implication of lipotoxicity to cytokinetic failure.

Initially, to investigate the effects of lipotoxic stress on cell cycle progression, we treated exponentially growing HeLa cells with different concentrations of palmitate. After 24-h treatment the cells were harvested and analyzed by FACS. Results showed that palmitate induced a dramatic $\mathrm{G} 2 / \mathrm{M}$ cell cycle arrest in a dose-dependent manner (Figure 1A). To discriminate $\mathrm{G} 2$ and $M$ phases, we further performed an M-index assay by immunostaining palmitate-treated cells against a mitosis marker Histone H3 (phosphor-S10), and results revealed a significant Mphase arrest by the palmitate treatment (Supplementary information, Figure S1).

Interestingly, we observed binucleate cell accumulation upon palmitate treatment with immunofluorescence microscopy (Figure 1B). The percentages of binucleate cells were counted and increased in a palmitate dose-dependent manner (Figure 1C). Since HeLa cell is a tumorous cell line, two non-tumor cell lines, NIH-3T3 and $\mathrm{C} 2 \mathrm{C} 12$, were also subjected to palmitate treatment, and we observed similar binucleate cell accumulation and a significant G2/ $M$ arrest in these two cell lines (data not shown), indicating that palmitate-induced cytokinetic failure might be a general phenomenon in mammalian cells.

To determine whether the binucleate cell formation is due to cytokinetic failure, cytokinesis of synchronized HeLa cells was monitored by time-lapse microscopy.
Cytokinesis is the final stage of eukaryotic cell division. During this stage, the mitotic cells use an actomyosinbased contractile ring that is attached to the plasma membrane to create a cleavage furrow after anaphase. This furrow then advances inwards to partition the mitotic cell into two daughter cells [3]. Our results showed that in control cells, when the cleavage furrow was formed, it quickly moved inwards and then an intracellular bridge, called the midbody, emerged within a few minutes (Figure 1D 'Control' and Supplementary information, Movie S1). While in the presence of palmitate, most of the cells that had cleavage furrow formation showed incomplete constriction of the contractile ring and then the furrows regressed (Figure 1D 'Palmitate' and Supplementary information, Movie S2). Notably, we also observed cells with no obvious furrow initiation under palmitate treatment (data not shown). Thus, the time-lapse study demonstrated that the palmitate-induced binuclear formation was due to a severe impairment of cytokinesis rather than cell-cell fusion.

RhoA, a small GTPase, plays a central role in cytokinesis control [4]. Inhibition of RhoA activity by chemical inhibitors or molecular genetic manipulation resulted in cytokinetic failure [5]. To elucidate the molecular mechanism by which palmitate impairs cytokinesis, we examined whether RhoA activity was suppressed under palmitate treatment by a biochemical pull-down assay (see Materials and Methods in Supplementary information, Data S1). The results demonstrated that RhoA activity was inhibited by palmitate in a dose- and time-dependent manner (Figure 1E and 1F). Furthermore, as previous reports show that active RhoA is highly concentrated at the site of contractile ring formation [6], we examined RhoA localization in cytokinetic cells by immunofluorescence microscopy. The results showed that RhoA was concentrated at the cleavage furrow in the control cells, whereas no similar aggregation of RhoA was observed in the palmitate-treated cells (Figure 1G). Therefore, we conclude that RhoA inhibition is an important factor that contributes to palmitate-induced cytokinetic failure.

However, neither wild-type RhoA nor constitutively 
A

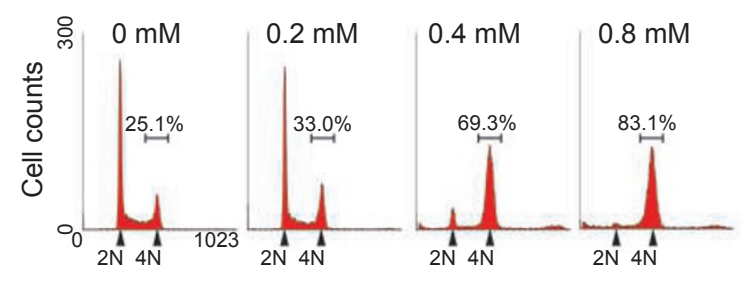

C

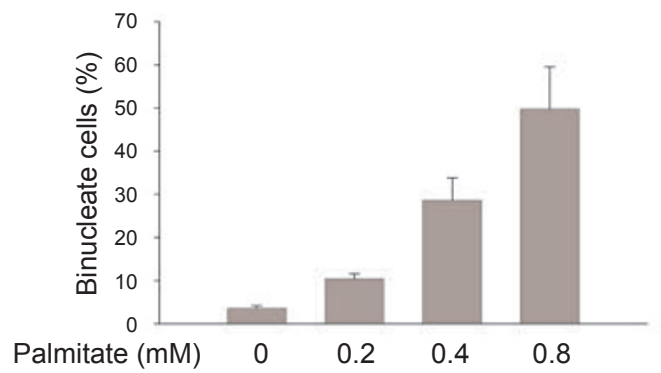

D

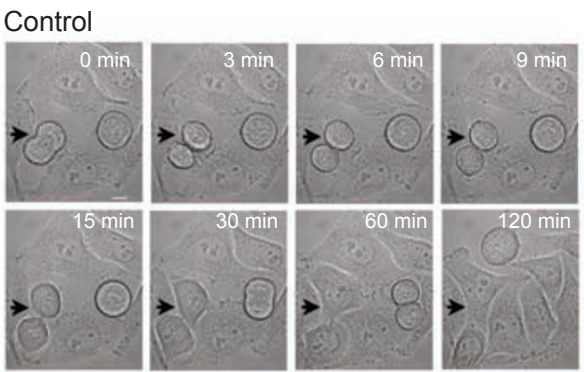

E

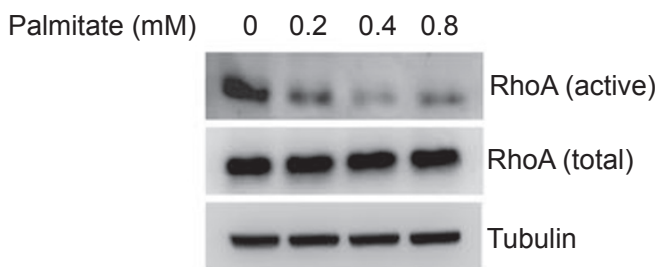

$\mathrm{F}$

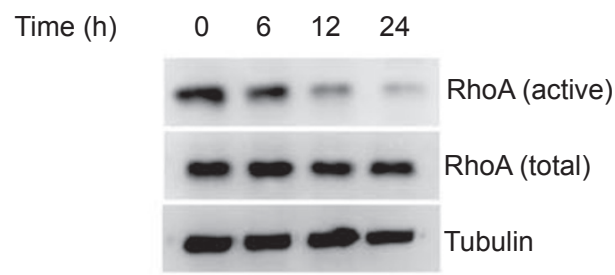

B
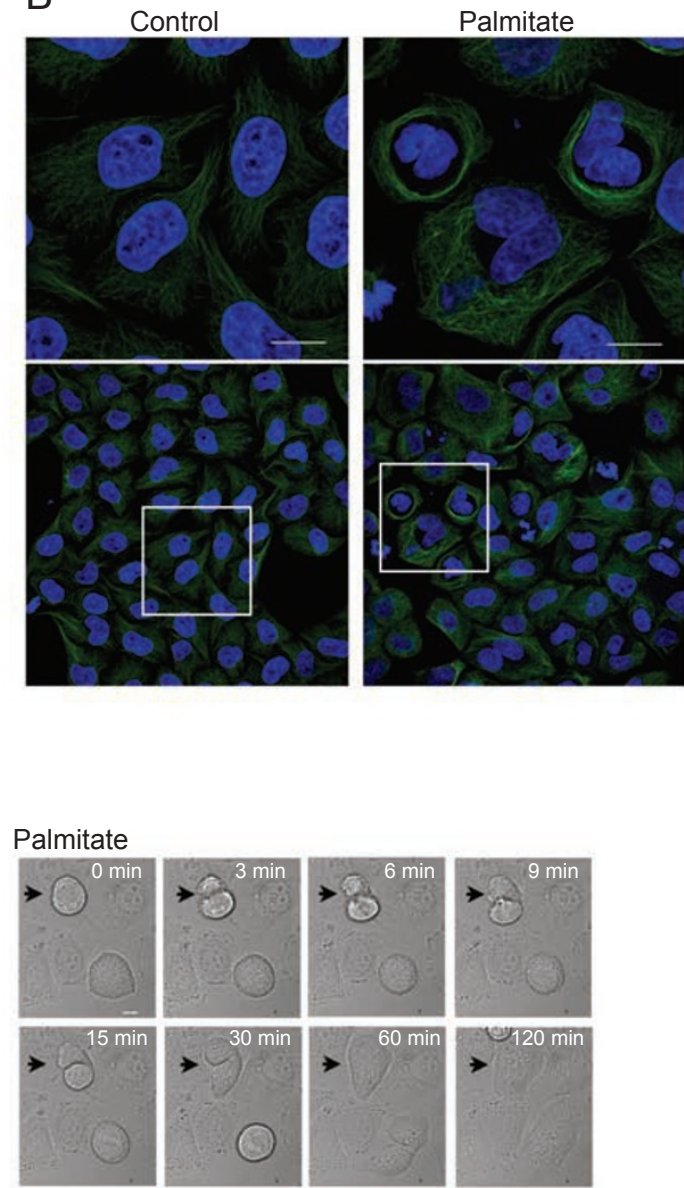

G

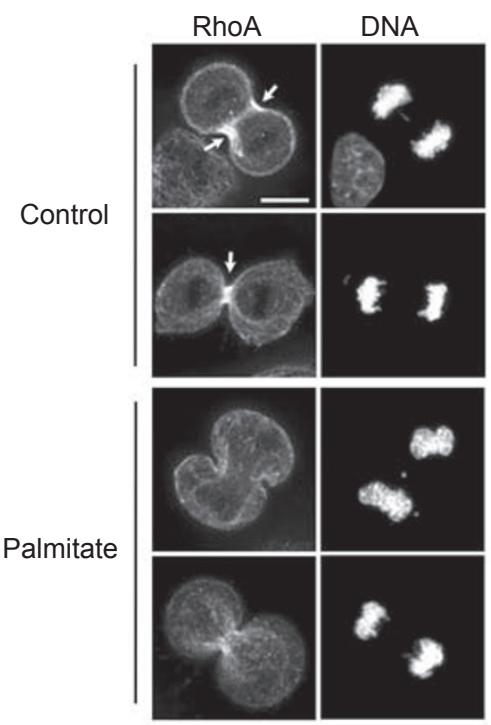

active RhoA (Q63L) was able to rescue palmitate-induced G2/M arrest (Supplementary information, Figure S2), indicating that the palmitate-induced cytokinetic impairment is a complex molecular event that must involve some other important molecules or pathways besides the reduced RhoA activity. In fact, we observed that the microtubule cytoskeleton, which is essential for RhoA activation in cytokinesis [7], was disorganized under palmi- 
tate treatment (Supplementary information, Figure S3). In addition, we found that two palmitoylation inhibitors, 2-bromopalmitate and cerulenin, and the mono-unsaturated fatty acid, oleate, could protect palmitate-treated cells from impaired cytokinesis with recovery of the suppressed RhoA activity (Supplementary information, Figure S4), implying that protein palmitoylation might be involved in the palmitate-induced cytokinetic failure. The role of lipids in cytokinesis has been recognized in some recent literatures [8-10]. Palmitate, as a free fatty acid, may potentially interfere with the function of these critical lipids, thereby inhibiting cytokinesis. Further studies are needed to analyze all these possibly impaired molecules or pathways under palmitate treatment. More importantly, it would be of significance to investigate whether the cytokinesis of dividing cells in vivo was impaired in patients of obesity and diabetes.

In conclusion, our data show that palmitate is able to induce cyokinetic failure in association with inhibition of RhoA activity, suggesting that cytokinetic failure is a possible defect derived from lipotoxicity.

\section{Acknowledgments}

This work was supported by the National Basic Research Program of China (973 Program) (2006CB503900 and 2006CB910703), grants from the National Natural Science Foundation of China (30821065), a grant from the Knowledge Innovation Program of the Chinese Academy of Sciences KSCX1YW-02, and a grant from Science and Technology Commission of Shanghai Municipality 07dz05907.

Jianhua Zhang ${ }^{1}$, Ying Yang ${ }^{1}$, Jiarui $\mathrm{Wu}^{1,2}$

${ }^{1}$ Key Laboratory of Systems Biology, State Key Laboratory of Molecular Biology, Institute of Biochemistry and Cell Bi- ology, Shanghai Institutes for Biological Sciences, Chinese Academy of Sciences, 320 Yue-Yang Road, Shanghai 200031, China: ${ }^{2}$ Hefei National Laboratory for Physical Sciences at Microscale and School of Life Sciences, University of Science \& Technology of China, Hefei, Anhui 230027, China

Correspondence: Jiarui $\mathrm{Wu}$

Tel: +86-21-54921128; Fax: +86-21-54920787

E-mail:wujr@sibs.ac.cn

\section{References}

1 Unger RH. Lipid overload and overflow: metabolic trauma and the metabolic syndrome. Trends Endocrinol Metab 2003; 14:398-403.

2 Brookheart RT, Michel CI, Schaffer JE. As a matter of fat. Cell Metab 2009; 10:9-12.

3 Barr FA, Gruneberg U. Cytokinesis: placing and making the final cut. Cell 2007; 131:847-860.

4 Glotzer M. The molecular requirements for cytokinesis. Science 2005; 307:1735-1739.

5 Piekny A, Werner M, Glotzer M. Cytokinesis: welcome to the Rho zone. Trends Cell Biol 2005; 15:651-658.

6 Yonemura S, Hirao-Minakuchi K, Nishimura Y. Rho localization in cells and tissues. Exp Cell Res 2004; 295:300-314.

7 Bement WM, Benink HA, von Dassow G. A microtubuledependent zone of active RhoA during cleavage plane specification. J Cell Biol 2005; 170:91-101.

8 Kanazawa T, Nakamura S, Momoi M, et al. Inhibition of cytokinesis by a lipid metabolite, psychosine. J Cell Biol 2000; 149:943-950.

9 Saul D, Fabian L, Forer A, Brill JA. Continuous phosphatidylinositol metabolism is required for cleavage of crane fly spermatocytes. J Cell Sci 2004; 117:3887-3896.

10 Field SJ, Madson N, Kerr ML, et al. PtdIns(4,5)P2 functions at the cleavage furrow during cytokinesis. Curr Biol 2005; 15:1407-1412.

(Supplementary information is linked to the online version of the paper on the Cell Research website.)

Figure 1 Palmitate impairs cytokinesis with suppressed RhoA activity. (A) Exponentially growing HeLa cells were treated with different concentrations of palmitate for $24 \mathrm{~h}$ and then analyzed by FACS. The percentages of G2/M phase were shown as indicated. Arrowheads indicate $2 \mathrm{~N}$ and $4 \mathrm{~N}$ DNA contents. (B) Merged confocal images of HeLa cells treated with palmitate (0.4 $\mathrm{mM}$ ) for $24 \mathrm{~h}$ and detected by immunostaining against tubulin (green) and DAPI staining of nuclei (blue), with high magnification at the top panels and low magnification at the bottom panels. Scale bar $=10 \mu \mathrm{m}$. (C) The percentages of binucleate cells were calculated at the indicated palmitate concentrations. More than 200 cells under each concentration were counted. Values are mean \pm SD from three independent experiments. (D) Thymidine-synchronized HeLa cells were incubated with control (left panel) or $0.4 \mathrm{mM}$ palmitate (right panel) for $6 \mathrm{~h}$ and then were monitored by time-lapse differential interference contrast microscopy. Images at indicated time points are shown. Arrows indicate the dynamics of a cellular furrow of cytokinesis. (E) RhoA activity was measured using the GST-RBD pull-down approach. When HeLa cells were treated with different doses of palmitate for $12 \mathrm{~h}$, active RhoA proteins were pulled down by GST-RBD beads, subjected to immunoblot analysis, and shown as 'RhoA (active)'. 'RhoA (total)' represents the total RhoA in whole cell lysates. A tubulin blot was performed as loading control. (F) HeLa cells were incubated with $0.4 \mathrm{mM}$ palmitate for the indicated times, and RhoA activity was measured as described above. (G) Confocal images showing RhoA localization in cytokinetic HeLa cells. The cells were treated without ('Control') or with $0.4 \mathrm{mM}$ palmitate ('Palmitate'), fixed with $10 \%$ TCA, and then immunostained against RhoA (left panels). DNA was stained with DAPI (right panels). Typical cells were shown. Arrows indicate concentrated RhoA localization at cleavage furrows. Scale bar $=10 \mu \mathrm{m}$. 